\title{
Solar dynamo in two-layer medium
}

\author{
Helen Popova \\ Faculty of Physics, M.V.Lomonosov Moscow State University \\ Leninskie Gory, Moscow, Russia \\ email: popovaelp@hotmail.com
}

\begin{abstract}
We studied the problem of the behavior of the magnetic field in the case of two-layer medium. We included of the meridional circulation in this model and investigated the influence of the meridional circulation on the nature of distribution and configuration of the dynamo-waves.
\end{abstract}

Keywords. waves, turbulence, MHD

\section{Introduction}

The cycles of solar magnetic activity associated with the action of the solar dynamo mechanism that is based on the combined effect of the differential rotation and alphaeffect. Such representation provides a solution in the form of oscillating waves of toroidal field, extending from middle latitudes to the equator. Scheme of the dynamo was proposed in the fundamental work by Parker (1955). The toroidal magnetic field is generated from the poloidal field by the differential rotation occurring in the solar convective zone. The reverse transformation of toroidal into poloidal field arises due to the absence of mirror symmetry in the convection occurring in the rotating body. In Parker (1955) it assumed that the generation of a dynamo occurs in one spherical shell, where the alpha-effect and differential rotation acting together. This scheme gives the duration of the cycle smaller than actually observed (Kuzanyan \& Sokoloff (1995)). Accounting for the meridional circulation provided the necessary lengthening of the cycle (Popova et al. (2008), Popova \& Sokoloff (2008), Popova (2009)).

However, consideration of a single-layer medium is described by one-way flow of matter and it does not allow describing return of matter. Parker suggested a way to describe an interface dynamo with dynamo generators distributed in two radial layers, using for each layer equations that are very similar to that of the Parker (1955) migratory dynamo Parker (1993). We developed a WKB method for the asymptotic solution of the corresponding dynamo equations. We consider two-layer medium, in which the layers have oppositely directed movement of substances and different diffusion coefficients. In this work we studied the problem of the behavior of the magnetic field in the case of two-layer medium with meridional circulation.

\section{Basic Equations}

We included the meridional flows in each layer of Parker equations

$$
\begin{aligned}
& \frac{\partial B}{\partial t}+\frac{\partial(V B)}{\partial \theta}=\beta \Delta B, \quad \frac{\partial A}{\partial t}+V \frac{\partial A}{\partial \theta}=\alpha B+\beta \Delta A \\
& \frac{\partial b}{\partial t}+\frac{\partial(v b)}{\partial \theta}=D \cos \theta \frac{\partial a}{\partial \theta}+\Delta b, \quad \frac{\partial a}{\partial t}+v \frac{\partial a}{\partial \theta}=\Delta a
\end{aligned}
$$


for a dynamo with the $\alpha$-effect present in one radial layer and shear of differential rotation present in the other. Here $\beta$ is the ratio of the diffusion coefficients in first and second layers, $B(r, \theta, t), b(r, \theta, t)$ are the corresponding toroidal magnetic fields in these layers, and $A(r, \theta, t), a(r, \theta, t)$ are proportional to the corresponding toroidal components of the vector potential (which determines the poloidal magnetic field). Here $V(\theta), v(\theta)$ are the meridional flows in the respective layers, $D$ is the dynamo number. Parker assumed the differential rotation to dominate in one layer and to vanish in the other, and, conversely, the $\alpha$-effect to prevail in the second layer and to vanish in the first.

Following Parker we prescribe $r=0$ for the radial boundary between two layers and use boundary conditions:

$$
b=B, \quad a=A, \quad \frac{\partial b}{\partial r}=\beta \frac{\partial B}{\partial r}, \quad \frac{\partial a}{\partial r}=\frac{\partial A}{\partial r} .
$$

In view of the symmetry conditions $\alpha(-\theta)=-\alpha(\theta), V(-\theta)=-V(\theta)$ the above system of equations can be considered in only one (e.g., the northern) hemisphere using antisymmetry (dipolar symmetry) or symmetry (quadrupolar symmetry) conditions at the equator.

We obtained Hamilton-Jacobi equation for Eqs. (2.1) and (2.2) by a method similar to the method described in Popova et al. (2010).

Remind that we represent the solution of Eqs. (2.1) and (2.2) in the form of waves travelling in the $\theta$-direction with an appropriate parametrization of the $r$-dependence:

$$
\begin{array}{ll}
B=\mu e^{i D^{1 / 3} S \theta+\gamma D^{2 / 3} t-i D^{1 / 3} m_{1} r}, & A=\left(\nu+D^{2 / 3} \nu_{1} r\right) e^{i D^{1 / 3} S \theta+\gamma D^{2 / 3} t-i D^{1 / 3} m_{1} r}, \\
a=\zeta e^{i D^{1 / 3} S \theta+\gamma D^{2 / 3} t-i D^{1 / 3} m_{2} r}, & b=\left(\chi+D^{-2 / 3} \chi_{1} r\right) e^{i D^{1 / 3} S \theta+\gamma D^{2 / 3} t-i D^{1 / 3} m_{2} r} .
\end{array}
$$

Here $\gamma, \nu, \nu_{1}, \varsigma, \chi, \chi_{1}, m_{1}, m_{2}$ are slowly varying functions of $\theta$ and $S(\theta)=\int k d \theta$.

$S$ is similar to the action in quantum theory and $k=S^{\prime}$ corresponds to the wave vector or impulse, which is complex in our case. The complex quantity $\gamma$ determines the eigenvalue, with its real part giving the growth rate and its imaginary part the duration of the activity cycle.

Substituting the desired form of solution in Eqs. (2.1) and (2.2) and using boundary conditions we obtained Hamilton-Jacobi equation for eqs. (2.1) and (2.2):

$$
\begin{aligned}
& \left(\beta \sqrt{-(\gamma+i V k) / \beta-k^{2}}+\sqrt{-\gamma-i v k-k^{2}}\right)\left(\sqrt{-(\gamma+i V k) / \beta-k^{2}}+\right. \\
& \left.+\sqrt{-\gamma-i v k-k^{2}}\right)=-\frac{4 \hat{\alpha} k}{i \beta \sqrt{-\gamma-i v k-k^{2}} \sqrt{-(\gamma+i V k) / \beta-k^{2}}} .
\end{aligned}
$$

For simplicity, we assume that the meridional circulation does not depend on latitude. Ways to study Hamilton-Jacobi equations with the meridional circulation are similar to the methods described in Popova (2009).

\section{Results}

In result we obtained the ratio of the diffusion coefficients affect to duration activity cycle. The growth of the intensity of the meridional flow of matter slows the spread of the dynamo waves. Minimum of the magnetic solar activity may occur in the case of large intensity of the meridional circulation in both layers, as well as substantial differences in physical characteristics between the layers. 


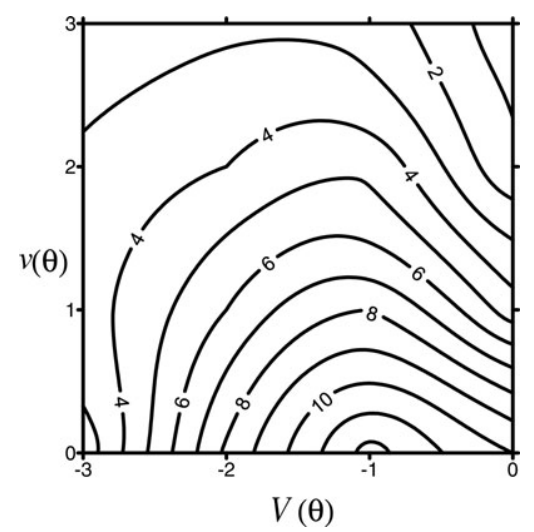

Figure 1. The ratios of $\beta, V(\theta)$, and $v(\theta)$ are for case when the duration of cycle is equal 11 years

Fig. 1 presents case when the meridional circulation is directed against spread of the dynamo waves in layer where differential rotation acting. Herewith, the meridional circulation is directed along spread of the dynamo waves in the layer where differential rotation acting. $V(\theta)$ is the meridional circulation in the layer with alpha-effect acting; $v(\theta)$ is the meridional circulation in the layer with differential rotation acting; isolines are beta. The ratios of $\beta, V(\theta)$, and $v(\theta)$ are for case when the duration of cycle is equal 11 years. To cycle duration was 11 years, as we can see from the figure, it must either increase the intensity of the meridional circulation in both layers or an increase $\beta$.

The more the meridional motion of matter is intense in the outer layer in comparison with the inner layer, the less $\beta$ can be to achieve the 11-years cycle. If the meridional flows in the inner layer of a substance is stronger than in the external layer it will require greater value $\beta$ to achieve the 11-years cycle than in the previous case.

If the values of $V(\theta)$ and $v(\theta)$ are interchanged the dependence of the cycle duration of $\beta$ will not be changed.

For $V(\theta)=v(\theta)=0$ the minimum of the solar activity ( $\sim 100$ years $)$ is achieved in case when $\beta \approx 30$. If $\beta$ tends to infinity the velocity of the dynamo wave spreading tends to zero. For $V(\theta) \neq v(\theta) \neq 0$ the minimum of the solar activity ( $\sim 100$ years) is achieved in case when either $\beta \approx 10$ and difference in the values of the velocities of the meridional flow from the different layers can be several tens or the order of $\beta$ is several tens and difference in the values of the velocities can be about 10 .

Obtained results results can be compared with observation data for $\beta, V(\theta)$, and $v(\theta)$.

Financial support from RFBR under grant 07-02-00127, 10-02-09688 and 09-02-01010 is acknowledged. Author is grateful to D. Sokoloff and M. Artyushkova for useful discussion.

\section{References}

Parker, E. N. 1955, Astrophys. J., 122, 293-314.

Kuzanyan, K. M. \& Sokoloff, D. D. 1995, Geophys. Astrophys. Fluid Dyn., 81, 113-129.

Popova, H. P., Reshetnyak, M. Yu., \& Sokoloff, D. 2008, Astron. Rep., 52, 157-163.

Popova, E. P. \& Sokoloff D. 2008, Astron. Nachr., 329, 766-768.

Popova, E. P. 2009, Astron. Rep., 53, 863-868.

Parker, E. N. 1993, Astrophys. J., 408, 707-719.

Popova, H. P., Artyushkova M. E., \& Sokoloff, D. 2010, Geophys. Astrophys. Fluid Dyn., ID: 507201 (in print). 